\section{The computational options}

\section{Christof Koch}

Neural and Brain Modeling. By Ronald J. MacGregor. Academic: 1988. Pp.643. Hbk $\$ 89, £ 56$; pbk $\$ 35, £ 22$.
IT HAS become very fashionable in the past few years to investigate various aspects of the nervous system from a theoretical point of view. Indeed 'experimental neuroscience' (until recently, quite simply 'neuroscience') has acquired a twin, 'computational neuroscience'.

This new field is characterized by two generally complementary but sometimes conflicting approaches. The 'bottom-up' strategy starts with a reasonably complete description of the system under study in terms of simple biophysical or neurophysical variables and attempts to follow its evolution in response to stimuli, usually on the basis of differential equations. The most successful instance of such a model is Hodgkin and Huxley's analysis in 1952 of the initiation and propagation of action potentials in terms of time- and voltagedependent membrane conductances. Today, the same technique - combined with the dendritic cable theory developed by W. Rall - is being applied to study the electrical properties of single identified neurons with their extended dendritic trees and dozens of membrane conductances. Similar detailed models are being elaborated at the neuronal network level, for instance in the case of a handful of neurons in the mollusc Tritonia or with thousands of neurons in the mammalian cerebellum or olfactory cortex.

In contrast, the 'top-down' strategy, popularized by D. Marr's influential book Vision, stems from the realization that it is best first to analyse complex informationprocessing systems, whether natural or artificial, at the abstract or "computational' level, well divorced from the hardware. At this level of analysis, any system, whether silicon or carbon based, will deal in a similar manner with the problem at hand, for instance with the reconstruction of the third dimension (depth) from two shifted, two-dimensional images. The two systems will differ as to how this is actually achieved in practice. In recent times, the limitations of a pure bottom-up approach, as practised by most neurophysiologists, and the pure top-down approach, as advocated by researchers in artificial intelligence, have been rejected in favour of a combination of both.

Although several books have appeared over the past years epitomizing the computational approach (most recently D. Rumelhart, J. McClelland and the PDP Research Group's Parallel Distributed Processing), no up-to-date work extols the virtues (and the methods) of modelling neuronal hardware at the dendrite, single neuron or neuronal network level. Thus, MacGregor's Neural and Brain Modeling will find an expectant audience.

The first 200 pages provide an overview of models, past and present, drawn primarily from the pages of Biological Cybernetics and Journal of Theoretical Biology. This survey includes accounts of Hodgkin and Huxley and their intellectual spawn; the application of cable theory to passive and active branched structures; models of the oculomotor system, the cerebellum, the visual, auditory and motor cortices; models of the electroencephalogram and epilepsy; models of learning and memory in neural networks; and the information-processing paradigm. There are no divas or stars for MacGregor though. The good, the bad and the ugly everybody seems to deserve his couple of lines, with little regard to whether their idea proved to be dead on arrival or resulted in a worthwhile advance in our understanding, and the models are always "powerful", "coherent", "elegant" and "exciting". Some theories, long out of fashion with both the neurobiological and the modelling community, are given plenty of space, for instance the fluid mechanical models of Beurle in the $1950 \mathrm{~s}$ and of Griffith in the 1960s in which neural differential equations representing spatiotemporal spread of neuroelectric activity. Other models receive scant mention, as for instance W. Reichardt's integrative approach towards understanding the visual system of the flies at the cellular, system and theoretical levels.

Ultimately, the usefulness of any abstract, mathematical model of reality has to be judged by its explanatory and predictive power. Specifically, what impact did the neural and brain models discussed in the book have on biophysics, neurophysiology or psychophysics? What is the relevance of these theories to experimental neuroscience? Fatally, MacGregor avoids these issues.

The second part of the book is organized around a number of annotated FORTRAN programs (including 180 pages of code; but at least we are spared flow charts), together with instructions, examples and exercises, enabling the user to model a large variety of neuronal systems, from a single-node neuron, to more sophisticated models with dendritic trees and active conductances, to systems of neuronal populations. MacGregor even includes a program to study the propagation of action potentials in branched axonal trees, an important and almost untouched area of research. This collection clearly represents the fruits of many years of effort. But although the range of phenomena which can be simulated numerically is impressive, the execution of these simulations is less so. For instance, little space is given to the seminal issue of the proper numerical technique to use for solving particular types of differential equations. The use of parallel networks were studied by formulating

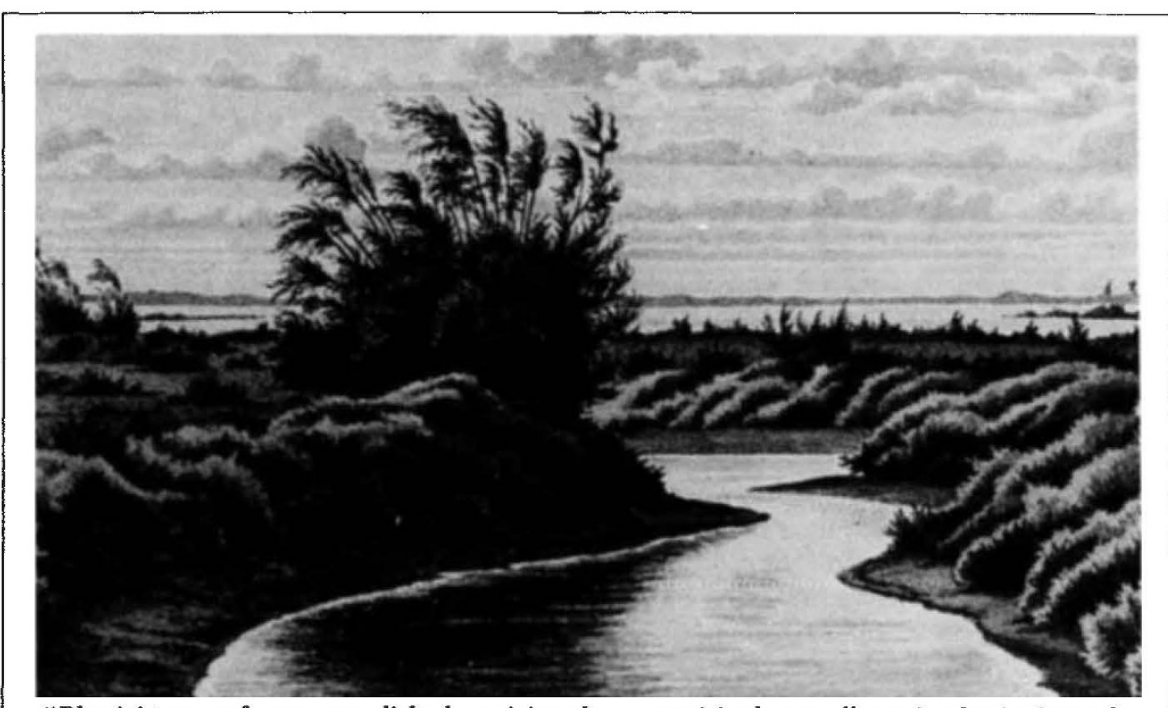

"Physicists are often accomplished musicians but, surprisingly, excellence in physics is rarely matched with creative ability in the visual arts" - so runs the jacket blurb to The Physicist as Artist: The Landscapes of Pierre Duhem, selected and introduced by Stanley L. Jaki, from which this detail of En Camargue (1899) is taken. Duhem, who died in 1916, was a prominent theoretical physicist, philosopher and historian of science, and produced over 400 landscapes many of which are reproduced in the book. Publisher is Scottish Academic Press, price is $£ 25$. 